\title{
The Application of Discourse Analysis in English Language Translation
}

\author{
Rongle Yao \\ English Department, School of Public Fundamentals, Jiangsu Vocational College of Medicine, \\ Yancheng, Panzhihua, 224006, China
}

Keywords: Discourse analysis; Translation; Application countermeasure

Abstract: With the continuous development of society, the method of discourse analysis is gradually changing, and the original surface description has turned to a deeper level of development. Stylistic, linguistic and physical analysis are three important ways in discourse analysis. This paper focuses on the three aspects of the relationship between the three and the role of discourse analysis.

\section{Introduction}

Discourse analysis is a branch of linguistics. It is a comprehensive and systematic understanding, analysis and evaluation of articles from the discourse structure. It uses discourse as a unit to interpret words and phrases around the whole content of discourse and analyze personality. And the reason of the event, summed up the central thinking and writing skills, but also pay attention to the cultural background knowledge and other related knowledge involved in the article.

The study of discourse analysis originated in the 1960s, when the concept of cohesion mainly refers to the function of linking sentences through certain words or grammatical features in the discourse. The famous British linguists Halliday and Hasan in 1976 The publication of Cohesion in English laid the foundation for textual cohesion theory. Since then, more and more people have been engaged in discourse analysis in just a few decades, which shows that people are aware of the analysis of discourse in foreign language learning. Great role, this impact can involve listening, reading comprehension, translation, writing and many other aspects. This article will use reading comprehension as an example to explain the theory and application of discourse analysis.

Discourse is a semantic whole realized by sentences. The sentences constituting a discourse are structurally connected to each other and semantically coherent. According to the theory of Halliday and Hasan in Cohesion in English, the cohesive device of English sentences can be divided into five categories: reference, substitution, ellipsis, conjunction, and lexical cohesion. In general, the first four are usually classified as Grammatical Cohesion. That is to say, the means of cohesion can be simply divided into two categories: grammatical cohesion and lexical cohesion. Among them, grammatical cohesion includes referencing, substituting, omitting and connecting words; Lexical Cohesion includes recurrence and co-occurrence. 


\section{The concept of discourse analysis}

The definition of discourse can be described as diverse. Halliday and Hasan believe that "text" refers to any spoken or written paragraph in linguistics that is not limited in length and constitutes a unified whole. Quirk et al. believe that: "text is a language with appropriate appropriateness in practical use.” One of the earliest scholars in China to study English discourse analysis is Huang Guowen, who pointed out that text usually refers to a series of words. The language of the paragraph or sentence. It can be a speech or an article. Discourse must be coherent and grammatical. At the same time, there must be a certain logical relationship between the discourse and the sentence. It is generally believed that the boundaries of discourse analysis should not be less than a single sentence, so most linguists believe that discourse analysis should be super-syntactic analysis. In summary, discourse is a unit larger than a sentence, and it is a language with a clear organization and a logical structure.

\section{Discourse analysis skills under the theory of discourse analysis}

Traditional discourse analysis translation emphasizes the understanding of surface structure such as word meaning and grammar, but it ignores sentences and sentences, paragraphs and paragraphs or external and internal exercises throughout the article. It lacks understanding of the whole article and hinders the translator's reading comprehension ability and independent analysis ability have been improved, and the translator's translation level has also been greatly affected. Discourse analysis can make up for this shortcoming. It emphasizes the understanding of the whole content, and then obtains the overall impression of the article. Then, with some analytical skills and clues, find sentences and sentences, paragraphs and paragraphs, paragraphs and chapters. The inter-connected relationship has a deep understanding of the discourse at the micro level.

\subsection{Pay attention to the society and enrich the readers' cultural background knowledge}

When translating, the translator has a rich background knowledge, which makes it easier to correctly understand and analyze the discourse. Translation practice research shows that only readers can link their own background knowledge with the information of the article to achieve a good understanding. However, our translators have insufficient knowledge in all aspects, so they have narrow knowledge. They often influence the understanding of the article and even distort the original intention of the author because of the limitations of their own cultural knowledge during translation. Reduced translation efficiency.

\subsection{Predicting the main content of the discourse based on the title or picture of the article}

When the article is translated, if we predict the content to be translated in advance, then the translator will become much easier to translate. However, if we don't know anything about the content of the article beforehand, then it will be very difficult to translate. Therefore, before the translator translates the article, he or she must first look at the clues of its title, picture or icon.

\subsection{Find key sentences or topic sentences}

Our translators know that the topic (The Top Sentence) also basically understands the main content of this or the whole article, because the topic sentence is the core of the text paragraph. In general, the layer unfolding of paragraphs is centered around the central sentence. Sometimes the central sentence is at the beginning of each paragraph, and sometimes the central sentence is at the 
end of each paragraph. Therefore, when the translator wants to translate the content, it is necessary to quickly find the central sentence, so that the main content of each paragraph and the theme of the whole article can be quickly grasped. After finding the topic of the article, it is easy to speculate on the approximate meaning of the strange words that appear in the translated content.

\subsection{Familiar writing style of discourse}

Although some discourses have no new words, it is difficult for some translators to understand. As you know, there are usually three structural patterns in English discourse: the first is the "problem-solve" model. In such an article, it is usually written in accordance with the "problem analysis - conclusion (countermeasure)", "phenomenon - consequences - recommendations", "contradictions - intensification - solution" and other routines. The second is the "general-special" mode. This kind of thinking about writing articles usually lays out articles with "phenomenon analysis - conclusions". The third is the "analog-contrast" mode. This way of writing is usually "phenomenon - other people's point of view - author's point of view" - things - advantages and disadvantages - conclusions. At the same time, it should be noted that paragraphs also have their own patterns [2]. This model is usually: top sentence, developing sentence, transitional sentence, concluding sentence. So, once the reader understands the pattern of writing, in the process of discourse analysis, you can easily grasp the author's writing context and understand the language easily. Article.

\subsection{Familiar with language cohesion and coherence}

In general, grammar and vocabulary can achieve language articulation on the surface structure of the discourse. Therefore, in writing teaching, teachers should teach students about the connection, so that students can write a structural, logical and contextual connection, just like a chain, a ring of closure. However, the means of cohesion is a necessary but insufficient condition for generating discourse. If cohesion does not necessarily produce discourse, only the deep semantic coherence in the discourse can make the article have a true adhesion and form a paragraph [5]. Therefore, coherence is also the principle to be followed by sentence segments. Coherence is the semantic association of texts. It exists at the bottom of discourse, and it is an invisible network of discourse. Only by attaching importance to coherence can the translated words be organized into a whole sentence that the translator can understand.

\section{The cohesive and coherent relationship}

When translating English and Chinese passages and texts, coherence is the extent to which a chapter is perceived as a whole rather than a series of irrelevant statements. Coherence is a useful whole for texts, rather than a kind of sensible discourse in the use of English language translation. This paper is an excellent essay on the use of discourse in English language translation. It has a certain reference and guidance for writing about coherent papers, and better realizes the coherence of semantics.

Cohesion is a chapter feature, coherence is a reader's evaluation of the chapter. The coherence of discourse should withstand the semantic connection between sentences and the logical reasoning of pragmatic environment. Therefore, discourse coherence not only includes the cohesion of the internal role of discourse, but also the cohesion between discourse and context. The basic criterion for a coherent discourse is that its role forms a whole and is associated with context. Cohesion is objective, theoretically able to be easily identified, and coherence is subjective, and the degree of coherence in the text will vary from reader to reader. The premise of cohesion is the logic and 
coherence of thinking, and coherence is an important guarantee for the success of communication. Cohesion is the external form of the text, and coherence is the inherent logical connection of the text. Cohesion is the tangible X-network of discourse, which is the semantic relationship between discourse surface structure forms. Coherence is the invisible X-network of discourse, which is the semantic or functional connection of deep texts [4].

\section{The application of discourse analysis in translation}

\subsection{Combine discourse and understand the original text}

Understanding is a prerequisite for translation. Translators can only translate in a certain context, analyze the discourse, determine the meaning of the entire discourse, and then determine the exact meaning of each sentence and each word. Discourse analysis can help the translator understand the original text from the following aspects: First, it can help the translator to determine the theme of a paragraph and grasp its general meaning. There is often a theme in the discourse that runs through the text and serves as a cohesive text. The second is to help the translator determine the meaning of the word and thus the meaning of the sentence and the text. Using the principle of discourse analysis, according to the characteristics of textual cohesion, the relationship between each sentence and each word is determined, so as to determine the exact meaning of a word and a phrase in the discourse. The third is to help the translator clearly refer to the relationship.

The pronouns in English are used more. Through careful discourse analysis, the translator can clearly understand the referential relationship in the original text. The fourth is to help the translator determine the omission components in some sentences. The omission in English rarely corresponds to the omission in Chinese; when translating from English to English, it is often necessary to supplement the omission component, which requires discourse analysis to find out these components [6].

\subsection{Analyze the discourse and grasp the context}

After a general understanding of the original text, it is necessary to clarify the relationship between the various sentences in the original text and the various actions described, which is also the deepening of the above understanding of the original text process. By analyzing the discourse, one can determine the logical connection between the sentences. In English, the logical connection between sentences is often marked by logical contact, indicating the semantic connection between sentences and sentences. However, it is sometimes difficult to determine logical relations by looking at logical connections. To determine the connection of sentences, it must be analyzed from the entire discourse. The second is to clarify the sequence of actions described in the discourse. In English, the order of actions is sometimes expressed only by the form of verbs and body. When translating, it is necessary to analyze which time and body in the text to determine which action is in front and which action is in the back. Third, it is possible to clarify the arrangement of information in sentences or texts, and to determine the main information, so as to grasp the focus of the text. The information arrangement of English discourse has a central principle at the end of the sentence, that is, the focus is on the end of the discourse, and this should be noted when translating.

\subsection{Arranging translations, smooth and smooth}

Translation not only has a process of understanding, but also a process of expression. As with understanding, expressions also use discourse analysis to accurately reproduce the meaning of the original text with a smooth and fluent translation. This requires a discourse analysis of both the 
original text and the translation. It is necessary to pay full attention to the differences between the two languages of English and Chinese, to get rid of the shackles of English cohesive means, and to make the translations coherent by using the cohesive means of Chinese habits.

Register analysis. "Registry analysis" begins with the analysis of discourse from the context of discourse formation. Because language is a social phenomenon, it is a meaningful interaction between people in a specific social situation, so language is not only vocabulary, grammar, phoneme system and structure, but connected language events. The behavior of the participants and their environment. "Regular analysis" is to analyze the use of language from the context and function of language. Through "locale analysis," we can know what context the text is produced in and what kind of relationship exists between the form and function of the language.

Discourse analysis and its role in translation are still to be further explored in many aspects. Translators are not too embarrassed, but intend to make a rough outline from the big part to show the discourse analysis in text translation and its translation. The important role [1].

\section{Conclusion}

Through the application of discourse analysis skills and discourse analysis in English translation, the following conclusions can be drawn. In English text translation, attention should be paid to the translation of these methods by the translation, according to the Chinese and English language text structure and expression habits. Combining the original texts and analyzing the discourses to grasp the key points and arrange the translations to be smooth and smooth, making the discourse easier for the reader to understand. Discourse analysis is deeply valued by scholars and translators both at home and abroad. Only by learning the discourse analysis can we achieve the ideal translation effect.

\section{References}

[1] Black Yuqin. On the role of text analysis mode in reading comprehension and teaching. Foreign Language Teaching. Vol.3 (2014) No.15, p. 136-150.

[2] Ma Bosen. Discourse Analysis Mode in Reading Teaching. Foreign Language Teaching and Research. Vol. 3 (2016) No.22, p. 22-25.

[3] Zhu Dongjiang, Chen Mei. Discourse Analysis and Text Translation Studies. Journal of Yancheng Teachers College (Humanities and Social Sciences Edition). Vol. 3 (2015) No.12, p. 11-15.

[4] Wang Zhengping. Discourse Analysis and English Reading Teaching. Journal of Xianning University. Vol. 1 (2015) No.11, p. 12-14.

[5] Mao Ronggui. An Analysis of Translations under the Perspective of Discourse Consciousness. Foreign Language Teaching. Vol. 12 (2013) No.3, p. 44-48.

[6] Zhang Junxi. Discussion on Schema Theory and Discourse Analysis Theory: Also, on Its Enlightenment to English Reading Teaching. Education Theory and Practice. Vol. 25 (2015) No.11, p. 122-124. 